\title{
Efficacy of infliximab in neuro-Behçet's disease presenting with isolated longitudinally extensive transverse myelitis
}

\author{
Ryo Kuroda $\cdot$ Junko Suzuki $\cdot$ Mizuho Muramatsu • \\ Asami Tasaki · Mariko Yano • Noboru Imai • \\ Masahiro Serizawa • Masahiro Kobari
}

Received: 8 August 2013/Revised: 5 October 2013/Accepted: 8 October 2013/Published online: 1 November 2013

(C) The Author(s) 2013. This article is published with open access at Springerlink.com

\section{Dear Sirs,}

Behçet's disease (BD) is a relapsing systemic inflammatory disorder of unknown etiology, showing common characteristics of oral and genital ulcers and uveitis. Several prospective studies have shown that neurological complications of BD, generally called neuro-Behçet's disease (NBD), occur in approximately 5-14\% of patients with BD [1-3]. In NBD, the frequency of spinal cord involvement is $8-14 \%[4,5]$.

Use of tumor necrosis factor (TNF)-alpha antagonist therapy has been described for NBD [6-8]. Nevertheless, reports of NBD with spinal cord involvement describing the efficacy of anti-TNF-alpha therapy are scarce [9]. We describe herein a case of NBD with longitudinal myelitis refractory to standard therapy that was successfully treated using infliximab, a TNF-alpha antagonist.

A 43-year-old Japanese man was admitted to our hospital with acute paralysis of both legs, and bowel and bladder incontinence. He had presented with headache at 33 years old. Recurrent oral ulceration, genital ulceration, and positive pathergy reaction were identified, and he had

R. Kuroda $(\bowtie) \cdot$ A. Tasaki $\cdot$ M. Yano · N. Imai ·

M. Serizawa $\cdot$ M. Kobari

Department of Neurology, Japanese Red Cross Shizuoka Hospital, 8-2 Ohte-machi, Aoi-ku, Shizuoka 420-0853, Japan e-mail: ryokrd@gmail.com

\section{J. Suzuki}

Department of Neurology, National Hospital Organization Utano Hospital, Kyoto, Japan

M. Muramatsu

Departments of Rheumatology, Japanese Red Cross

Shizuoka Hospital, Shizuoka, Japan fulfilled the International Behçet's disease Study Group criteria [10].

On admission, examination showed spastic paraparesis with hyperreflexia in the lower extremities, including pathological reflexes. Results from blood tests were unremarkable, and negative results were obtained for both HLA-B51 and anti-aquaporin-4 antibody. Spinal magnetic resonance imaging (MRI) showed abnormally bright signals on T2-weighted imaging in the T12 spinal cord. Cerebrospinal fluid (CSF) analysis demonstrated increased lymphocytes $\left(23\right.$ cells $\left./ \mathrm{mm}^{3}\right)$ and protein $(78 \mathrm{mg} / \mathrm{dl})$. CSF interleukin (IL)-6 was markedly elevated $(214 \mathrm{pg} / \mathrm{ml}$, normal $<4.0 \mathrm{pg} / \mathrm{ml}$ ). CSF IgG oligoclonal banding was negative. We diagnosed NBD with isolated extensively transverse myelitis. Treatment with intravenous methylprednisolone (m-PSL) at $1,000 \mathrm{mg} /$ day for 3 days improved symptoms. A maintenance dose of $15 \mathrm{mg} /$ day of oral PSL was administered.

Seven months later, his gait gradually deteriorated and he became paraparetic. Intravenous m-PSL pulse therapy was administered, but his disease remained active. A persistent high CSF IL-6 concentration $(60.2 \mathrm{pg} / \mathrm{ml})$, which exceeded the critical threshold $(>20 \mathrm{pg} / \mathrm{ml}$ ) for progression of neurological manifestations in chronic progressive NBD [11], indicated that his clinical course had proceeded to the secondary progressive type. The patient was administered oral methotrexate (6-10 mg/week) for 4 months, but little improvement was seen and his walk remained slow and spastic.

Twelve months after the first admission, he suddenly became totally paraplegic and was rehospitalized. Sensations of pinprick, temperature, and vibration were totally absent in both legs. MRI of the thoracic spine showed an abnormally increased longitudinal T2 signal from T6 to T10 (Fig. 1a, b). Contrast-enhanced T1-weighted imaging 

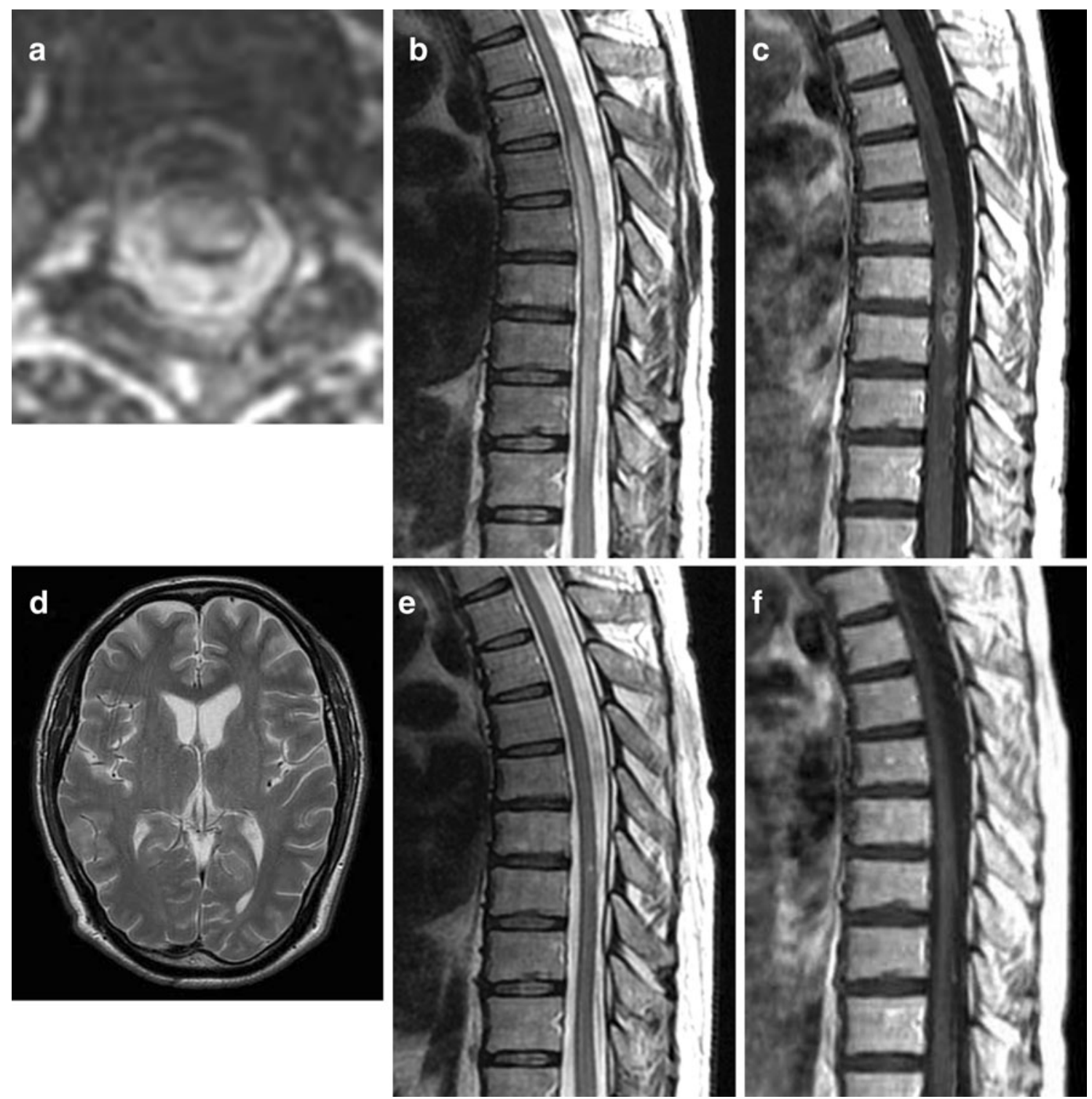

Fig. 1 Spine magnetic resonance imaging (MRI) before $(\mathbf{a}-\mathbf{c})$ and after (e, f) infliximab therapy and cranial MRI (d). a Axial T2weighted image showing transverse myelitis. b Sagittal T2-weighted image showing abnormally increased longitudinal T2 signal with mild enlargement from T6 to T10. c Sagittal contrast-enhanced T1-

revealed partial enhancement of the lesion (Fig. 1c). Cranial MRI had no abnormal finding (Fig. 1d). The CSF level of IL-6 was extremely high $(424 \mathrm{pg} / \mathrm{ml})$. Despite receiving IV m-PSL pulse therapy, no clinical improvement was obtained, and he could neither bend his knees nor transfer to a wheelchair by himself. Intermittent urethral catheterization was needed due to severe urinary dysfunction.

Infliximab was started $(5 \mathrm{mg} / \mathrm{kg}$ at weeks 0,2 , and 6 , and every 8 weeks thereafter). A dramatic improvement in his severe spasticity was noticed within $24 \mathrm{~h}$ after initiating the first infusion and remained stable throughout the observation period. Repeat MRI of the spine performed weighted image revealing partial enhancement of the lesion. d Cranial MRI had no abnormal finding. e Sagittal T2-weighted image showing reduced longitudinal abnormal signal. f Sagittal contrast-enhanced T1-weighted image revealing no enhancement lesion

2 weeks later revealed reduction of longitudinal abnormal signals on T2-weighted imaging (Fig. 1e). The lesions with contrast enhancement disappeared (Fig. 1f). CSF IL-6 was reduced to $18.3 \mathrm{pg} / \mathrm{ml}$. At 4 weeks, the patient was able to stand unaided, and use of intermittent catheterization was stopped. Over the subsequent 6 months, he has maintained clinical improvement (detailed clinical course in Table 1), and has achieved the ability to walk while holding tables. No adverse effects were seen during the observation period.

We have confirmed the efficacy of infliximab in a refractory NBD patient with isolated spinal cord 
Table 1 Clinical course of symptoms and CSF findings and the treatment regimen

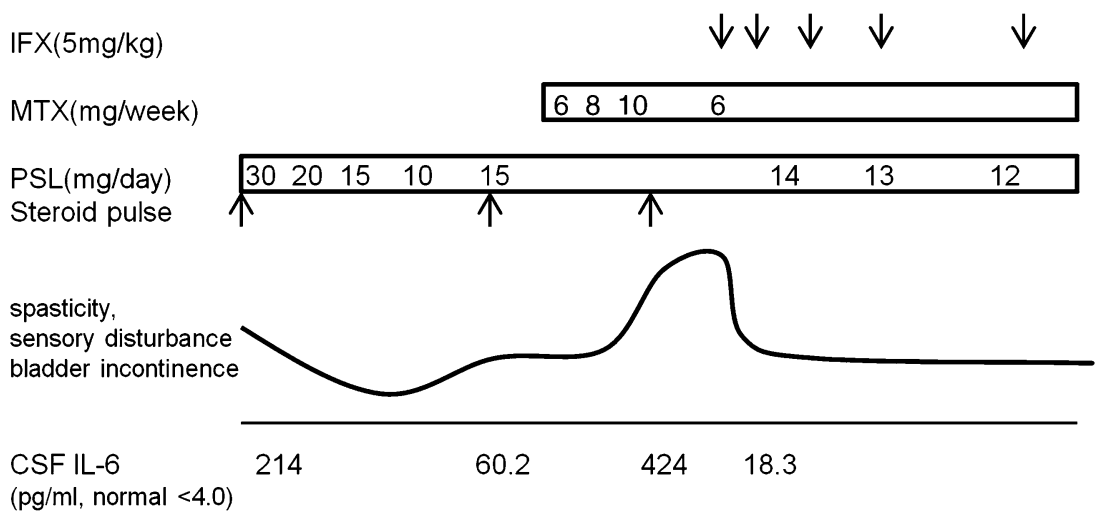

PSL prednisolone, MTX methotrexate, IFX infliximab, $C S F$ cerebrospinal fluid, $I L-6$ interleukin-6

involvement. A previous study suggested that NBD patients with spinal cord involvement show worse prognosis than those with other types of NBD [12]. In that report, over $70 \%$ of the 24 patients showed a primary or secondary progressive course, and eight patients died, despite administration of steroid and immunosuppressants. Appropriate therapeutic intervention is thus needed to prevent the development of irreversible neurological sequelae.

Inflammation in $\mathrm{BD}$ is thought to be mediated by cytokines, particularly TNF-alpha, which is located upstream of cytokine networks, including the pathway that produces IL-6 [13]. Some articles on TNF-alpha antagonist therapy for steroid/immunosuppressant-refractory NBD involving other neurological sites have been published, and most have reported satisfactory clinical responses to therapy without severe side effects [6-8]. However, only one report has described NBD with spinal cord involvement that was successfully treated using anti-TNF-alpha therapy [9].

To the best of our knowledge, this represents the first published report of NBD with spinal cord involvement successfully treated using infliximab according to a detailed cytokine profile. We confirmed reduction in CSF levels of IL-6 after infliximab treatment, along with diminished disease activity. This finding supports previous reports about other clinical phenotypes of NBD [13, 14].

No consensus has been reached regarding the optimal timing to start infliximab therapy. The present case showed a number of poor prognostic factors, including spinal cord involvement, multiple attacks, progressive course, and high CSF levels of IL-6. We suggest earlier initiation of infliximab therapy in the presence of abnormal CSF IL-6 concentrations and other poor prognostic factors.
In conclusion, this case supports the view that infliximab is a potent therapeutic option for refractory NBD cases with spinal cord involvement.

Conflicts of interest The authors declare that they have no conflicts of interest.

Ethical standard We declare that we got appropriate informed consent from the patient and respect the confidentiality and anonymity of our patient. We avoid harm to our participant.

Open Access This article is distributed under the terms of the Creative Commons Attribution License which permits any use, distribution, and reproduction in any medium, provided the original author(s) and the source are credited.

\section{References}

1. Al-Araji A, Sharquie K, Al-Rawi Z et al (2003) Prevalence and patterns of neurological involvement in Behçet's disease: a prospective study from Iraq. J Neurol Neurosurg Psychiatry 74:608-613

2. Serdarouglu P, Yazici H, Ozdemir C et al (1989) Neruological involvement in Behçet's syndrome-a prospective study. Arch Neurol 46:265-269

3. Ashjazadeh N, Borhani Haghighi A, Samangooie S et al (2003) Neuro-Behçet's disease: a masquerader of multiple sclerosis. A prospective study of neurologic manifestations of Behçet's disease in 96 Iranian patients. Exp Mol Pathol 74:17-22

4. Akman-Demir G, Serdarouglu P, Tasci B et al (1999) Clincal patterns of neurological involvement in Behçet's disease: evaluation of 200 patients. Brain 122:2171-2182

5. Ideguchi H, Suda A, Takeno M et al (2010) Neurological manifestations of Behçet's disease in Japan: a study of 54 patients. J Neurol 257:1012-1020

6. Pipitone N, Olivieri I, Padula A et al (2008) Infliximab for the treatment of neuro-Behçet's disease: a case series and review of the literature. Arthritis Rheum 59:285-290

7. Al-araji A, Saip ASS, Constaninescu C et al (2010) Treatment of neuro-Behçet's disease with infliximab. An international multicentre case-series of 18 patients. Clin Exp Rheumatol 28:S119 
8. Borhani Haghighi A, Safari A, Nazarinia MA et al (2011) Inflizimab for patients with neuro-Behçet's disease: case series and literature review. Clin Rheumatol 30:1007-1012

9. Coulter I, Huda S, Baborie A et al (2012) Longitudinally extensive transverse myelitis as the sole presentation of neuroBehçet's disease responding to infliximab. J Spinal Cord Med 35:122-124

10. International study group for Behçet's disease (1990) Criteria for diagnosis of Behçet's disease. Lancet 335:1078-1080

11. Hirohata S, Isshi K, Oguchi H et al (1997) Cerebrospinal fluid interleukin-6 in progressive neuro-Behçet's syndrome. Clin Immunol Immunopathol 82:12-17
12. Yesilot N, Mutlu M, Gungor O et al (2007) Clinical characteristics and course of spinal cord involvement in Behçet's disease. Eur J Neurol 14:729-737

13. Kikuchi H, Asako K, Takayama M et al (2008) Effect of infliximab in progressive neuro-Behçet's syndrome. J Neurol Sci 272:99-105

14. Matsui T, Ishida T, Tono T et al (2010) An attack of acute neuroBehcet's disease during the course of chronic progressive neuroBehçet's disease: report of two cases. Mod Rheumatol 20:621-626 